\title{
Avaliação do Custo do Implante Programado de Múltiplos Stents em Mais de uma Intervenção Percutânea em Pacientes com Doença Coronária Multiarterial
}

\author{
Alessandro Pina Pedroso1, George Cesar Ximenes Meireles ${ }^{1,2}$, Luciano Mauricio de Abreu Filho', \\ Antonio Artur da Cruz Forte ${ }^{1}$, Marcos Kiyoshi Sumita ${ }^{1}$, Desidério Favarato ${ }^{3}$, José Del Carmen Solano Aliaga ${ }^{1}$
}

RESUMO

Fundamentos: O Sistema Único de Saúde estabelece que a angioplastia coronária com implante de dupla prótese intraluminal não deverá exceder o total de $20 \%$ das angioplastias realizadas, resultando na necessidade de escalonar a maioria dos procedimentos nos pacientes com doença coronária multiarterial. O objetivo deste estudo foi comparar os custos do escalonamento estabelecido pelo SUS para implante de stent ao planejamento de implante de stent considerado ideal pela equipe de cardiologia intervencionista, em pacientes com doença coronária multiarterial, com indicação de implante de mais um stent por procedimento. Método: Os custos foram comparados em uma série consecutiva de 100 pacientes com doença multiarterial com indicação de revascularização completa por intervenção coronária percutânea com o implante de dois ou mais stents por paciente. As coronariografias foram previamente avaliadas pela equipe de cardiologia intervencionista, que indicou o escalonamento considerado ideal para o tratamento das estenoses coronárias em cada paciente. Em seguida, foram realizados os procedimentos de acordo com os critérios do SUS e os custos das duas estratégias comparados. O valor do procedimento de implante de um stent pela tabela SUS é de R\$4.298,00. Resultados: A idade média foi $61,5 \pm 10,7$ anos, $66 \%$ eram do sexo masculino, $43 \%$ eram diabéticos e $67 \%$ tinham angina estável. Ocorreu concordância em relação ao escalonamento estabelecido pelo SUS em 44\% e discordância em $56 \%$. Foram realizados 58 procedimentos a mais do que o considerado ideal pela equipe de cardiologia intervencionista, gerando um custo adicional de R\$131.298,00. Conclusões: $O$ escalonamento estabelecido pelo SUS, com-
SUMMARY

Cost Evaluation of Scheduled Multiple Stents Implantation in Multivessel Coronary Disease Patients

Background: The Brazilian Public Health System (SUS) establishes that coronary angioplasties with implantation of two stents must not exceed $20 \%$ of the total amount of angioplasties performed, resulting in the necessity of staged procedures in the majority of the patients with multivessel coronary disease. The objective of this study was to compare the costs of the staged stent implantation policy, imposed by SUS, with the stent implantation approach considered ideal by the interventional cardiology team, in patients with multivessel coronary disease. Methods: Costs were compared in a consecutive cohort of 100 patients with multivessel disease with indication of complete revascularization by percutaneous coronary intervention. Coronary angiographies had been previously evaluated by the interventional cardiology team that had individualized the approach considered ideal for the treatment of each patient. Procedures were then performed in accordance to SUS multistage policy and the costs of the two treatment strategies were compared. Of note, a single stent implantation procedure costs $\mathrm{R} \$ 4,298.00$ according to the SUS price list. Results: Mean age was $61.5 \pm 10.7$ years, $66 \%$ were male, $43 \%$ diabetics and $67 \%$ had stable angina. Agreement in relation to the SUS staged procedures occurred in $44 \%$ and discordance in $56 \%$. An excess of 58 procedures was detected, generating an additional cost of $\mathrm{R} \$ 131,298.00$. Conclusions: The multistaged approach imposed by SUS increases the number of procedures for coronary stent

Hospital Stella Maris, São Paulo, SP

2 Hospital do Servidor Público Estadual, São Paulo, SP.

3 Instituto do Coração do Hospital das Clínicas da Faculdade de Medicina da Universidade de São Paulo, São Paulo, SP. Correspondência: George César Ximenes Meireles. Secretaria da Pós-Graduação do IAMSPE. Avenida Ibirapuera, 981, 6ํaㅁ andar, sala 601 - Vila Clementino - São Paulo, SP - CEP 04029-000. Tel.: (11) 5088-8452 / (11) 5088-8338 - E-mail: george.ximenes@terra.com.br Recebido em: 19/6/2007 • Aceito em: 10/8/2007 
parado ao considerado ideal pela equipe de cardiologia intervencionista, ocasiona um aumento no número de procedimentos de implante de stent coronário e, consequentemente, uma elevação de gastos públicos.

DESCRITORES: Contenedores. Revascularização miocárdica. Política de saúde. implantation in patients with multivessel disease and consequently increments the public expenses.

DESCRIPTORS: Stents. Myocardial revascularization. Health policy.

O objetivo deste estudo foi comparar os custos do escalonamento estabelecido pelo SUS para o implante de stent ao planejamento de implante de stent considerado ideal pela equipe de cardiologia intervencionista, em pacientes com doença coronária multiarterial com indicação de implante de mais de um stent por procedimento.

\section{MÉTODO}

Trata-se de registro unicêntrico, consecutivo, comparativo de custos do escalonamento de implante de stent estabelecido pelo SUS em relação ao escalonamento considerado ideal pela equipe de cardiologia intervencionista, em 100 pacientes com doença arterial coronária multiarterial com indicação de implante de dois ou mais stents coronários.

O protocolo de estudo foi aprovado pelo Comitê de Ética em Pesquisa da instituição e foi conduzido de acordo com os princípios da declaração de Helsinque. O termo de consentimento livre e esclarecido foi obtido de todos os pacientes.

As indicações dos procedimentos foram realizadas por cardiologistas clínicos. Assim, foram incluídos pacientes com doença coronária multiarterial, portadores de angina estável ou síndrome coronária aguda com indicação de revascularização completa percutânea, com o implante de dois ou mais stents coronários. Os critérios de inclusão angiográfica foram presença de lesões com porcentual de estenose em diâmetro $>50 \%$ em duas ou mais artérias coronárias nativas, com diâmetro de referência $\geq 2 \mathrm{~mm}$, passíveis de tratamento pela intervenção coronária percutânea.

Foram excluídos os pacientes com contra-indicação ao uso de aspirina e clopidogrel, pacientes submetidos à angioplastia primária e pacientes portadores de doença sistêmica crônica capaz de influenciar a sobrevida (insuficiência hepática ou renal crônica, neoplasia maligna, doença cerebrovascular e doença do tecido conjuntivo).

O sucesso angiográfico foi definido como a obtenção do porcentual de estenose em diâmetro menor que $20 \%$ com fluxo TIMI 3 e o sucesso clínico pela obtenção do sucesso angiográfico na ausência de eventos cardíacos maiores (morte cardíaca, infarto ou cirurgia de emergência). O diagnóstico de infarto pós-implante 
Pedroso AP, et al. Avaliação do Custo do Implante Programado de Múltiplos Stents em Mais de uma Intervenção Percutânea em Pacientes com Doença Coronária Multiarterial. Rev Bras Cardiol Invas 2007; 15(3): 249-254.

era feito quando: 1. houvesse surgimento de ondas $Q$ anormais, em duas derivações contíguas que explorassem a mesma parede, não presentes no eletrocardiograma inicial do paciente; 2 . houvesse elevação enzímica maior que três vezes o limite superior normal da creatinofosfoquinase e de sua fração MB.

As coronariografias foram previamente avaliadas pela equipe de Cardiologia Intervencionista, que indicou o escalonamento considerado ideal para o tratamento das estenoses coronárias em cada paciente. Em seguida, foram realizados os procedimentos de acordo com os critérios de escalonamento do SUS do uso de dupla prótese intraluminal arterial no mesmo procedimento, não excedendo $20 \%$ do total de angioplastias coronárias realizadas. Uma vez concluídos todos os tratamentos, foi comparada a diferença do número de procedimentos realizados e os previstos pela equipe de cardiologia intervencionista, bem como os custos das duas estratégias.

Para a análise de custos foram considerados os valores da Tabela SIH/SUS de $\mathrm{R} \$ 4.298,00$ e $\mathrm{R} \$ 6.332,83$ para o implante de uma e duas próteses intraluminal arterial, respectivamente, o que corresponde a um custo do procedimento de $\mathrm{R} \$ 2.263,77$ e do stent de $\mathrm{R} \$ 2.034,23$. Foi considerada a mesma quantidade de stents para as duas estratégias, pois o que as diferenciava era apenas o escalonamento. Então, para efeito de cálculos foi utilizado apenas o custo do procedimento.

As variáveis quantitativas foram apresentadas como médias e desvios-padrão; foram calculadas freqüências absolutas e relativas às variáveis qualitativas.

\section{RESULTADOS}

No período de junho de 2005 a fevereiro de 2007, foram incluídos 100 pacientes para revascularização completa pelo implante de stent coronário.

As características clínicas dos pacientes estão listadas na Tabela 1. A idade média foi $61,5 \pm 10,7$ anos, com predomínio do sexo masculino (66\%). Foi observado que $43 \%$ dos pacientes eram diabéticos, 11 haviam se submetido à CRM e a função ventricular esquerda média era normal.

$\mathrm{Na}$ análise angiográfica, observamos prevalência de pacientes com três $(52,9 \%)$ ou quatro $(24,4 \%)$ vasos e estenoses $\geq 50 \%$ em ramos marginais esquerdos (29,8\%), artéria coronária direita (23\%) e artéria descendente anterior (20\%) - Tabela 2.

Foram implantados 351 stents em 301 vasos $(1,16$ stent/vaso e 3,5 stents/paciente). Ocorreu sucesso clínico em 97,2\%, 97,1\% e 98,3\% e ocorreu um pseudoaneurisma, um IAM relacionado com oclusão de ramo diagonal pós-implante na ADA e um óbito por oclusão aguda pós-implante de stent na ACD, nos pacientes que se submeteram as primeiras, segundas e terceiras séries de angioplastias, respectivamente. A revascularização completa foi obtida em $95 \%$ dos pacientes. O tempo médio entre a $1^{\underline{a}}$ e a $2^{\underline{a}}$ angioplastias e entre a $2^{\text {a }}$ e a $3^{\text {a }}$ angioplastias foi $46 \pm 27,6$ e 45,6 $\pm 26,7$ dias, respectivamente, e não ocorreu dano maior na espera.

Ocorreu concordância em relação ao escalonamento estabelecido pelo SUS em 44\% e discordância em 56\% (Tabela 3 e Figura 1). Foram realizados 58

TABELA 1

Características clínicas dos pacientes

\begin{tabular}{lc}
\hline & $\mathbf{n = 1 0 0}$ \\
\hline Idade (anos) & $61,5 \pm 10,7$ \\
Sexo masculino & 66 \\
Tabagismo atual & 54 \\
Hipertensão arterial sistêmica & 79 \\
Hipercolesterolemia (>200 mg/dL) & 45 \\
Diabetes mellitus (>125 mg/dL) & 43 \\
Infarto do miocárdio prévio & 4 \\
Reestenose & 4 \\
Cirurgia de revascularização prévia & 11 \\
Angina estável & 67 \\
Síndrome coronária aguda sem supra ST & 23 \\
Síndrome coronária aguda com supra ST & 10 \\
Fração de ejeção do & \\
ventrículo esquerdo & $67 \pm 14,3 \%$ \\
\hline
\end{tabular}

TABELA 2

Número de vasos e artérias tratadas

\begin{tabular}{lc}
\hline $\mathbf{N}^{\circ}$ pacientes e vasos com estenose $\mathbf{3 0} \%$ & Total $(\mathbf{n})$ \\
\hline 28 pacientes com 2 vasos & 56 \\
52 pacientes com 3 vasos & 156 \\
18 pacientes com 4 vasos & 72 \\
1 paciente com 5 vasos & 5 \\
1 paciente com 6 vasos & 6 \\
Total & 295 \\
Artérias coronárias & Total $(\mathrm{n})$ \\
A. coronária direita $(*)$ & 68 \\
A. descendente anterior $(* *)$ & 59 \\
A. circunflexa & 26 \\
Ramos marginais $\left(^{* * *}\right)$ & 88 \\
Ramos diagonais & 26 \\
Ramos diagonalis & 2 \\
Ramo ventricular posterior & 14 \\
Ramo descendente posterior & 5 \\
Ponte de veia safena & 6 \\
Mamária esquerda - ADA & 1 \\
Total & 295
\end{tabular}

6 pacientes com 2 lesões no mesmo vaso: $* 1$ artéria com 2 lesões; ${ }^{* *} 4$ artérias com 2 lesões; ${ }^{* * *} 1$ artéria com 2 lesões. 
TABELA 3

Resultados dos procedimentos

\begin{tabular}{lccc}
\hline & $\mathbf{1}^{\mathbf{a}}$ - ATC & $\mathbf{2}^{\mathbf{a}}$ ATC & $\mathbf{3}^{\mathbf{a}}$ ATC \\
\hline № lesões tratadas & $120(40 \%)$ & $125(41,5 \%)$ & $56(18,5 \%)$ \\
№ stents & 149 & 141 & 61 \\
Resultado & & & $55(98,2 \%)$ \\
$\quad$ Sucesso & $117(97,5 \%)$ & $122(98,4 \%)$ & $1(1,8 \%)$ \\
$\quad$ Insucesso & $3(2,5 \%)$ & $3(1,6 \%)$ & $1(2,2 \%)$ \\
Complicações & - & $1(1 \%)$ & - \\
$\quad$ Óbito & - & - & - \\
IAM & $1(1 \%)$ & & - \\
Vasculares & & & \\
\hline
\end{tabular}

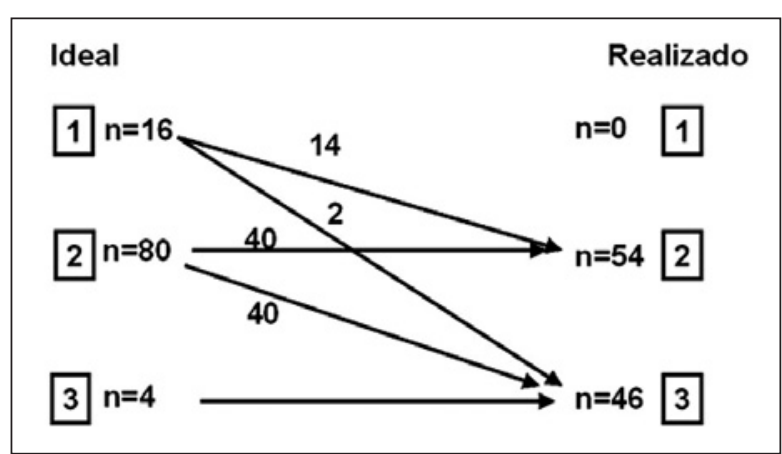

Figura 1 - Escalonamento proposto pela equipe de hemodinâmica/ escalonamento estabelecido pelo SUS.

procedimentos a mais do que o considerado ideal pela equipe de cardiologia intervencionista, gerando um custo adicional de R\$131.298,7.

Os critérios utilizados pela equipe de hemodinâmica para o escalonamento do implante de stents foram: dificuldade de acesso vascular, anatomia coronária desfavorável (por exemplo, intensa angulação proximal), lesões complexas (tipo C), insuficiência cardíaca ou renal e síndrome coronária aguda.

\section{DISCUSSÃo}

As vantagens da intervenção coronária percutânea em relação à CRM são o menor risco imediato de morbimortalidade cerebrovascular, pulmonar e renal, principalmente em pacientes idosos e em portadores de doença prévia destes sistemas; o tempo mais curto de convalescença e de internação hospitalar; o retorno precoce às atividades profissionais e menores custos hospitalares e após a alta ${ }^{16}$. Dados recentes, incorporando os avanços tecnológicos no implante de stents e nas CRM, demonstraram que os custos da hospitalização inicial e aos dois anos após o tratamento de pacientes com doença coronária multiarterial com múltiplos stents convencionais versus CRM, foram mais vantajosos para o grupo dos stents ( $27 \%$ de redução) a despeito de uma taxa maior de nova revascularização neste grupo ${ }^{17,18}$.

Embora a CRM permita revascularização mais completa, com a ICP podemos, deliberadamente, realizar revascularização anatomicamente incompleta, mas funcionalmente completa, baseando-se na avaliação não invasiva de viabilidade miocárdica, evitando assim o tratamento de vasos relacionados a áreas fibróticas sem miocárdio viável, e na avaliação invasiva nas estenoses consideradas angiograficamente moderadas com o uso da medida de reserva de fluxo fracionado do miocárdio e da ultra-sonografia intracoronária ${ }^{19,20}$.

A intervenção percutânea em pacientes multiarteriais apresenta também em relação à cirurgia a possibilidade do procedimento ser realizado de forma escalonada, na dependência da apresentação clínica, função renal, complexidade das lesões, função ventricular e acesso vascular. Pacientes com síndrome coronária aguda, freqüentemente, apresentam placas coronárias múltiplas complexas - aproximadamente 40\% dos pacientes com IAM apresentam placas ulceradas ou evidência de trombo em outros segmentos arteriais. A abordagem mais comum, nesses pacientes com doença multiarterial, é tratar a lesão "culpada" e tratar posteriormente as outras lesões em situação clinicamente mais estável. O tratamento de pacientes com doença multiarterial associada à disfunção ventricular, insuficiência renal ou lesões complexas também pode ser realizado de forma escalonada, diminuindo a exposição a raios- $X$, a quantidade de contraste radiológico e as complicações relacionadas ao procedimento $^{19}$.

A análise de impacto no orçamento visa auxiliar aos tomadores de decisão da Secretária de Atenção à Saúde do Ministério da Saúde, na tarefa de avaliar modificações na atual sistemática que estabelece que a angioplastia coronariana com implante de dupla prótese intraluminal arterial não deve exceder o total de $20 \%$ do total de angioplastias coronárias realizadas. No presente estudo, no grupo discordante desta sistemática foi proposto o implante de stents em ape- 
Pedroso AP, et al. Avaliação do Custo do Implante Programado de Múltiplos Stents em Mais de uma Intervenção Percutânea em Pacientes com Doença Coronária Multiarterial. Rev Bras Cardiol Invas 2007; 15(3): 249-254.

nas um tempo, em 16 pacientes, e foi realizado em dois tempos, em 14 pacientes (+ 14 procedimentos), e, em três tempos, em 2 pacientes (+ 4 procedimentos) e, em dois tempos, em 40 pacientes e foi realizado em 3 tempos (+40 procedimentos), totalizando 58 procedimentos a mais, o que gerou $\mathrm{R} \$ 2.263,77$ por procedimento, um custo adicional de $\mathrm{R} \$ 131.298,7$.

Ressaltamos que esta estratégia imposta pelo SUS não somente é antieconômica, como cerceia a liberdade do médico de escolher qual a melhor abordagem terapêutica para determinado paciente e, principalmente, expõe esses a mais procedimentos do que seria necessário, com seus riscos inerentes.

Segundo dados da Central Nacional de Intervenções Cardiovasculares (CENIC) da Sociedade Brasileira de Hemodinâmica e Cardiologia Intervencionista ${ }^{21}$, a prevalência de pacientes com doença coronária multiarterial que se submeteram a ICP aumentou de 40,6\%, em 1997, para $49,1 \%$, em 2005, e o número de vasos tratados por pacientes de 1,18 para 1,3 no mesmo período.

No ano de 2003, o SUS financiou a realização de 30.666 angioplastias coronarianas com implante de stent e de 19.909 cirurgias de revascularização do miocárdio, totalizando aproximadamente R\$281 miIhões em despesas ${ }^{22}$. Considerando que, atualmente, aproximadamente metade das intervenções coronárias é realizada para o tratamento de pacientes portadores de doença coronária multiarterial, podemos estimar que a economia sem a restrição imposta pelo SUS seja de, no mínimo, uma dezena de milhões de reais.

Apesar da conhecida superioridade dos stents farmacológicos em relação aos stents convencionais em reduzir a necessidade de novas revascularizações da lesão-alvo ${ }^{23-26}$, não foram utilizados esses stents neste estudo devido a sua não incorporação pelo SUS. Segundo dados da CENIC de 2005, a prevalência do uso de stents farmacológicos no Brasil nas intervenções coronárias percutâneas é de aproximadamente 17,5\%

Recentemente, foi realizada análise de impacto do stent farmacológico no orçamento do SUS e foi observado que o uso de stent farmacológico tem custo adicional comparativamente ao uso de stent convencional, no primeiro ano de utilização, no $\mathrm{SUS}^{27}$.

A pesquisa de novas abordagens e tecnologia é uma oportunidade para os membros da $\mathrm{SBHCl}$ contribuírem com os formuladores de políticas de saúde cardiovascular no processo de escolha entre as alternativas disponíveis, mensurando o benefício para cada unidade de custo e estimando o retorno para a sociedade da incorporação de novas modalidades diagnósticas e terapêuticas.

\section{CONCLUSÃO}

O escalonamento estabelecido pelo SUS comparado ao considerado ideal pela equipe de cardiologia intervencionista ocasiona aumento no número de procedimentos de implante de stent coronário e, conseqüentemente, uma elevação de gastos públicos.

\section{REFERÊNCIAS BIBLIOGRÁFICAS}

1. European Coronary Study Group. Long-term results of prospective randomized study of coronary artery bypass surgery in stable angina pectoris. Lancet. 1982;2(8309):1173-80.

2. Detre KM, Takaro T, Hultgren H, Peduzzi P. Long-term mortality and morbidity results of the Veterans Administration randomized trial of coronary artery bypass surgery. Circulation. 1985;72(6 pt 2):V84-9.

3. Abizaid AAC, Siqueira DA. Revascularização miocárdica em multiarteriais. Arq Bras Cardiol. 2005;84(3):202-5.

4. Coronary angioplasty versus coronary artery bypass surgery: the Randomized Intervention Treatment of Angina (RITA) trial. Lancet. 1993;341(8845):573-80.

5. King SB $3^{\text {rd }}$, Lembo NJ, Weintraub WS, Kosinki AS, Barnhart $\mathrm{HX}$, Kutner $\mathrm{MH}$, et al. A randomized trial comparing coronary angioplasty with coronary bypass surgery. Emory Angioplasty versus Surgery Trial (EAST). N Engl J Med. 1994;331(16):1044-50.

6. CABRI Trial Participants. First-year results of CABRI (Coronary Angioplasty versus Bypass Revascularization Investigation). Lancet. 1995;346(8984):1179-84.

7. The Bypass Angioplasty Revascularization Investigation (BARI) Investigators. Comparison of coronary bypass surgery with angioplasty in patients with multivessel disease. N Engl J Med. 1996;335(4):217-25.

8. Rodriguez A, Mele E, Peyregne E, Bullon F, Perez-Balino N, Liprandi MI, et al. Three-year follow-up of the Argentine Randomized Trial of Percutaneous Transluminal Coronary Angioplasty Versus Coronary Artery Bypass in Multivessel Disease (ERACI). J Am Coll Cardiol. 1996;27(5):1178-84.

9. Serruys PW, Unger F, Sousa JE, Jatene A, Bonnier HJ, Schonberger JP, et al. Comparison of coronary-artery bypass surgery and stenting for the treatment of multivessel disease. N Engl J Med. 2001;344(15):1117-24.

10. Rodriguez A, Bernardi V, Nava J, Baldi J, Grinfeld L, Martinez J, et al. Argentine Randomized Study: Coronary Angioplasty with Stenting versus Coronary Bypass Surgery in patients with Multiple-Vessel Disease (ERACI II): 30-day and oneyear follow-up results. ERACI II Investigators. J Am Coll Cardiol. 2001;37(1):51-8.

11. SoS Investigators. Coronary artery bypass surgery versus percutaneous coronary intervention with stent implantation in patients with multivessel coronary artery disease (the Stent or Surgery trial): a randomized controlled trial. Lancet. 2002;360(9338):965-70.

12. Rodriguez AE, Baldi J, Fernandez Pereira C, Navia J, Rodriguez Alemparte M, Delacasa A, et al. Five-year follow-up of the Argentine randomized trial of coronary angioplasty with stenting versus coronary bypass surgery in patients with multiple vessel disease (ERACI II). J Am Coll Cardiol. 2005;46 (4):582-8.

13. Hoffman SN, TenBrook JA, Wolf MP, Pauker SG, Salem DN, Wong JB. A meta-analysis of randomized controlled trials comparing coronary artery bypass graft with percutaneous transluminal coronary angioplasty: one- to eight-year outcomes. J Am Coll Cardiol. 2003;41(8):1293-304.

14. Centemero M, Sousa JE, Jatene AD, Paulista PP, Souza LCB, Sousa AGMR. Evolução clínica tardia de pacientes multiarteriais tratados por revascularização percutânea e cirúrgica. Rev Bras Cardiol Invas. 2007;15(1):35-43.

15. Ministério da Saúde. Secretaria de Atenção à Saúde. Departamento de Regulação, Avaliação e Controle. Manual do 
Pedroso AP, et al. Avaliação do Custo do Implante Programado de Múltiplos Stents em Mais de uma Intervenção Percutânea em Pacientes com Doença Coronária Multiarterial. Rev Bras Cardiol Invas 2007; 15(3): 249-254.

Sistema de Informação Hospitalar - Atualização. Volume I. Brasília:Editora do Ministério da Saúde; 2006.

16. Zago AC, Sousa AG, Sousa JE. Evolução da intervenção percutânea para o tratamento da doença coronária multiarterial. Arq Bras Cardiol. 2002;78(2):250-60.

17. Reynolds MR, Neil N, Ho KK, Berezin R, Cosgrove RS, Lager RA, et al. Clinical and economic outcomes of multivessel coronary stenting compared with bypass surgery: a singlecenter US experience. Am Heart J. 2003;145(2):334-42.

18. Nagle PC, Smith AW. Review of recent US cost estimates of revascularization. Am J Manag Care. 2004;10(11 Suppl):S370-6.

19. Zimarino M, Calafiore AM, De Caterina R. Complete myocardial revascularization: between myth and reality. Eur Heart J. 2005;26(18):1824-30.

20. Hannan EL, Racz M, Holmes DR, King SB $3^{\text {rd }}$, Walford G, Ambrose JA, et al. Impact of completeness of percutaneous coronary intervention revascularization on long-term outcomes in the stent era. Circulation. 2006;113(20):2406-12.

21. Sociedade Brasileira de Hemodinâmica e Cardiologia Intervencionista. Disponível em http://www.sbhci.org.br
22. Ministério da Saúde. Informações em saúde: assistência à saúde. Disponível em http://www.datasus.gov.br.

23. Morice MC, Serruys PW, Sousa JE, Fajadet J, Ban Hayashi E, Perin $M$, et al. A randomized comparison of a sirolimuseluting stent with a standard stent for coronary revascularization. N Engl J Med. 2002;346(23):1773-80.

24. Stone GW, Ellis SG, Cox DA, Hermiller J, O’Shaughnessy C, Mann JT, et al. A polymer-based paclitaxel-eluting stent in patients with coronary artery disease. $N$ Engl J Med. 2004;350(3): 221-31.

25. Babapulle MN, Joseph L, Belisle P, Brophy JM, Eisenberg MJ. A hierarchical Bayesian meta-analysis of randomized clinical trials of drug-eluting stents. Lancet. 2004;364(9434):583-91.

26. Serruys PW, Ong AT, Moriec MC, De Bruyne B, Colombo A, Macaya C, et al. Arterial Revascularization Therapies Study Part II: sirolimus-eluting stents for the treatment of patients with multivessel de novo coronary stent lesions. Eurointervention. 2005;1:147-56.

27. Araújo DV, Lima VC, Ferraz MB. Análise de impacto do stent farmacológico no orçamento do Sistema Único de Saúde. Arq Bras Cardiol. 2007;88(4):458-63. 